\title{
MIDPOINT TECHNICAL REPORT
}

to

\author{
DOE/OBES
}

for

\section{Contract DE-FG03-93ER 14313 \\ April 19, 1993}

by

Alan C. Tripp

University of Utah

\section{DISCLAIMER}

This report was prepared as an account of work sponsored by an agency of the United States Government. Neither the United States Government nor any agency thereof, nor any of their employees, makes any warranty, express or implied, or assumes any legal liability or responsibility for the accuracy, completeness, or usefulness of any information, apparatus, product, or process disclosed, or represents that its use would not infringe privately owned rights. Reference herein to any specific commercial product, process, or service by trade name, trademark, manufacturer, or otherwise does not necessarily constitute or imply its endorsement, recommendation, or favoring by the United States Government or any agency thereof. The views and opinions of authors expressed herein do not necessarily state or reflect those of the United States Government or any agency thereof. 
Improving the resolution of electromagnetic imaging requires that the forward solution be made as computational efficient as possible and that a-priori information be incorporated into the image in an efficient manner. In the past 6 months progress has been made in both aspects.

Appendix A contains a report of progress made in optimizing a three-dimensional (3D) integral equations forward modeling code which we will use for many of our imaging studies. Of particular note here is the coupling of the system iteration and group symmetry reduction methods of solution optimization. Both of these methods were discussed in earlier DOE reports and publications, but to date they have not been coupled in the present manner. We have also run extensive comparisons of the present code against independent codes to check numerical accuracy and computational speed. We find that the code agrees well with several other "bench mark" codes and that the various optimizations make the code very fast. We also have the option to run high conductivity contrast models with no change in basis functions, unlike other integral equation codes.

Appendix B contains a report of a technique for regularizing electric imaging by designing an array which optimizes the resolution of deviations from an a-priori model. We feel that such an approach could be used with profit for electromagnetic imaging, particularly in areas such as oil fields where a significant amount of a-priori information is already known. 


\section{Appendix A}

\section{Advances in 3D EM Modeling}

\section{A.1 Introduction}

Electromagnetic modeling of three dimensional conductivity structures using integral equations has been widely accepted in geophysics since its appearance in the early 1970s (Hohmann, 1971, 1975; Weidelt, 1975). Yet the quadratically growing requirements on computer storage and on the computation time in forming the scattering matrix, and the cubic growth of matrix factorization time with the number of discretization cells have remained the major obstacles for its application for about two decades.

To mitigate these computational problems using geometric model symmetries, Tripp and Hohmann (1984; see also Tripp, 1990, 1982) put forward a block diagonalization.1 method which reduces the scattering matrix to four block diagonal matrices and reducies the computation time for matrix formation by a factor of four. The method also reduces the storage requirement by a factor of 16 and the matrix factorization time by a factor of 16.

More recently Xiong (1992) introduced the method of system iteration which markedly reduces the matrix factorization time and storage requirements. In the method of system iteration a conductivity structure is divided into many substructures. The direct matrix inversion is applied to each substructure only, whereas the mutual interactions among the substructures are treated as due to equivalent sources. This method not only solves the problem of storage requirements, but also greatly reduces the computation time needed for the solution of the matrix equation. Furthermore, the method of system iteration also allows vector and/or parallel processing.

An obvious ploy is to combine these two methods. We will report on this combination in this appendix. 
We have also run many comparisons of the code as presently configured with other codes. This work is also presented here. We find that the present code, which includes group reductions (Tripp and Hohmann, 1984; Tripp, 1990, 1982), matrix system iteration (Xiong, 1992), and spatial symmetry reduction (Xiong \& Tripp, 1993) is very fast. In addition it agrees well with other "bench mark" codes. A spinoff of the care taken in Green's fucntion integration is that the code can be used without changing the basis functions for evaluating high conductivity contrast models.

For symmetric models the four block matrices obtained by group reduction are further partitioned into block submatrices. Thus we need only factorize directly one diagonal subblock-matrix of one of the four block matrices at a particular time. The system iteration, or block iteration, is then carried out for each quadrant.

\section{A.2 The Integral Equation Method}

For completeness we provide a brief descrption of the basic integral equation method. We shall follow the notation and discussions of Xiong (1992). The earth is assumed to be vertically anisotropic with a conductivity tensor $\underline{\sigma}_{n}$ of a diagonal form with the entries $\sigma_{h}, \sigma_{h}$, and $\sigma_{v}$, where $\sigma_{h}=\sigma_{h}^{\prime}+i \omega \epsilon_{h}$ and $\sigma_{v}=\sigma_{v}^{\prime}+i \omega \epsilon_{v}$ are the complex conductivities of the medium in the horizontal and the vertical directions, respectively.

The scattering current, denoted by $\mathbf{J}_{\boldsymbol{s}}$, within an arbitrary three-dimensional structure embedded in the anisotropic host is defined as

$$
\mathbf{J}_{\mathbf{s}}=\underline{\sigma}_{a} \cdot \mathbf{E},
$$

with $\underline{\sigma}_{a}=\sigma \underline{\delta}-\underline{\sigma}_{n}$, where $\underline{\delta}$ is a unit tensor, $\sigma$ the conductivity of the structure which we assume to be isotropic for simplicity, and $\mathbf{E}$ the total electric field in the structure.

The scattering current $\mathbf{J}$, within a structure $V$ satisfies the following Fredholm integral equation of the second kind:

$$
\underline{\Theta} \cdot \mathbf{J}_{s}(\mathbf{r})-\int_{V} \underline{G}^{E}\left(\mathbf{r} \mid \mathbf{r}^{\prime}\right) \cdot \mathbf{J}_{s}\left(\mathbf{r}^{\prime}\right) d v^{\prime}=\mathbf{E}_{n}(\mathbf{r}),
$$

with

$$
\underline{\Theta}=\left[\begin{array}{ccc}
\frac{1}{\sigma-\sigma_{h}} & 0 & 0 \\
0 & \frac{1}{\sigma-\sigma_{h}} & 0 \\
0 & 0 & \frac{1}{\sigma-\sigma_{v}}
\end{array}\right],
$$

where $E_{n}$ is the incident electric field in the absence of the 3D scatterer, and $\mathbf{G}^{E}$ the electric Green's tensor. Once $\mathbf{J}_{\mathbf{s}}$ is found from equation (A.2), the electromagnetic fields 
everywhere can be determined by the integration of the scattering current with corresponding Green's functions.

Equation (A.2) is usually discretized by dividing the structure $V$ into $M$ cells, which yields the matrix equation,

$$
[\Gamma]\left[J_{s}\right]=\left[E_{n}\right]
$$

with

$$
\underline{\boldsymbol{\Gamma}}_{m n}\left(\mathbf{r}_{m} \mid \mathbf{r}_{n}\right)=\delta_{m n} \underline{\Theta}_{m}-\int_{v_{n}} \underline{G}^{E}\left(\mathbf{r}_{m} \mid \mathbf{r}_{n}\right) d v
$$

where

$$
\delta_{m n}=\left\{\begin{array}{ll}
1, & m=n \\
0, & m \neq n .
\end{array} .\right.
$$

The coefficient matrix $\Gamma$ in equation (A.3) is usually refered to as the scattering matrix. It is also known as the impedance matrix (Tripp and Hohmann, 1984). The size of $\Gamma$ is $N$ by $N$ where $N=3 \times M$, since we have 3 components for each vector quantity. The $m$ in $\underline{\Theta}_{m_{0}}$ indicates that the conductivity $\sigma$ in $\underline{\Theta}$ is calculated at $\mathbf{r}_{m}$.

\section{A.3 Simultaneous execution of the method of sys- tem iteration and group reductions for con- trolled source problems}

\section{A.3.1 The method of system iteration}

The governing equation for the scattering current $\mathbf{J}$, within an inhomogeneity, equation (A.2), can be rewritten as

$$
\underline{\Theta} \cdot \mathbf{J}_{s i}(\mathbf{r})-\int_{V_{i}} \underline{G}^{E}\left(\mathbf{r} \mid \mathbf{r}^{\prime}\right) \cdot \mathbf{J}_{s i}\left(\mathbf{r}^{\prime}\right) d v^{\prime}=\mathbf{E}_{n}(\mathbf{r})+\sum_{j, j \neq i}^{m} \int_{V_{j}} \underline{\mathbf{G}}^{E}\left(\mathbf{r} \mid \mathbf{r}^{\prime}\right) \cdot \mathbf{J}_{s j}\left(\mathbf{r}^{\prime}\right) d v^{\prime}
$$

if we divide the structure $V$ into many smaller substructures, $V_{1}, V_{2}, \ldots$, and $V_{m}$. We are then able to solve for the scattering currents in all the substructures by an iterative approach. Equation (A.5) shows that we consider the mutual interactions among the substructures to be exciting sources to the $i$ th substructure.

From the viewpoint of matrix theory, equation (A.5) is equivalent to partitioning the scattering matrix $\Gamma$ into many block matrices, $\Gamma_{i j}$, and solving the matrix equation using a block iterative method as follows:

$$
\left[\begin{array}{cccc}
\Gamma_{11} & \Gamma_{12} & \ldots & \Gamma_{1 m} \\
\Gamma_{21} & \Gamma_{22} & \ldots & \Gamma_{2 m} \\
\cdot & \cdot & \cdot & \cdot \\
\Gamma_{m 1} & \Gamma_{m 2} & \ldots & \Gamma_{m m}
\end{array}\right]\left(\begin{array}{c}
J_{s 1} \\
J_{s 2} \\
\cdot \\
J_{s m}
\end{array}\right)=\left(\begin{array}{c}
\mathbf{E}_{n 1} \\
\mathbf{E}_{n 2} \\
\cdot \\
\mathbf{E}_{n m}
\end{array}\right)
$$


and

$$
\left[\Gamma_{i i}\right]\left[J_{o i}^{k+1}\right]=\left[E_{n i}\right]-\sum_{j=1}^{i-1}\left[\Gamma_{i j}\right]\left[J_{s j}^{k+1}\right]-\sum_{j=i+1}^{m}\left[\Gamma_{i j}\right]\left[J_{s j}^{k}\right],
$$

where $\mathrm{J}_{s i}^{k+1}$ is determined by matrix inversion. The diagonal matrices $\Gamma_{i i}$ can be factored using $U D U^{T}$ or $L U$ factorization, depending on whether or not they are symmetric. The factored matrices are then stored for use in the system iteration. This block iterative method is a generalization of the conventional point Gauss-Seidel method.

There are several advantages of the method as compared with direct solutions of equation (A.2), which include

- The reduced requirements on computer memory, since only the diagonal submatrices corresponding to each substructure need to be factorized directly.

- The system becomes more stable since we don't need to invert the whole matrix.

- The method saves computation time in solving the matrix equation since the system iteration converges rapidly.

- It is possible to use parallel computing.

\section{A.3.2 Group reductions}

If the 3D model possess the property of two-plane symmetry, then the scattering matrix $\Gamma$, or impedance matrix in the nomenclature of by Tripp \& Hohmann (1984), can be block diagonalized using a unitary matrix $U$ (Tripp \& Hohmann 1984). Let us rewrite equation (A.3) in a simpler form as

$$
\Gamma J_{s}=E_{n}
$$

By a similarity transform of the above equation we have

$$
\Gamma^{\prime} J_{s}^{\prime}=E_{n}^{\prime}
$$

with

$$
\begin{aligned}
\Gamma^{\prime} & =U \Gamma U^{T} \\
J_{s}^{\prime} & =U J_{s} \\
E_{n}^{\prime} & =U E_{n} .
\end{aligned}
$$

The block diagonalized matrix $\Gamma^{\prime}$ has the form of 


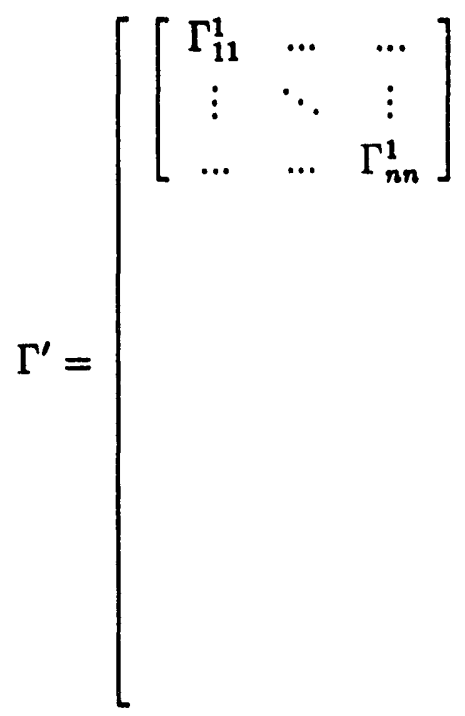

$$
\left[\begin{array}{ccc}
\Gamma_{11}^{2} & \ldots & \ldots \\
\vdots & \ddots & \vdots \\
\ldots & \ldots & \Gamma_{n n}^{2}
\end{array}\right]
$$$$
\left[\begin{array}{ccc}
\Gamma_{11}^{3} & \ldots & \ldots \\
\vdots & \ddots & \vdots \\
\ldots & \ldots & \Gamma_{n n}^{3}
\end{array}\right]
$$

and equation (A.9) reduces to four equations of smaller dimension:

$$
\begin{aligned}
& \Gamma^{1} J_{0}^{1}=E_{n}^{1} \\
& \Gamma^{2} J_{0}^{2}=E_{n}^{2} \\
& \Gamma^{3} J_{0}^{3}=E_{n}^{3} \\
& \Gamma^{4} J_{0}^{4}=E_{n}^{4}
\end{aligned}
$$

The dimension of the block matrices $\Gamma_{n n}^{1}$ etc. is $1 / 4$ of the original matrix $\Gamma$. Therefore, the storage requirement is reduced by a factor of 4 , and the computer core memory requirement is reduced by a factor of 16 since only one block matrix need be factorized at a time. The computation time for forming the block matrices is reduced by a factor of 4. The compu'ation time for factorizing the matrices is reduced by a factor of 16 .

\section{A.3.3 Simultaneous execution of system iteration and group reduction}

We may further block partition the matrices $\Gamma^{\prime}$ in equation (A.13) into many sub-blockmatrices as in equation (A.6) and apply the method of system iteration for each of the four equations in (A.14) through (A.17). In this way we reduce the storage requirements to one substructure of one quarter only as well as preserve all other advantages of the method of system iteration.

The method of system iteration has been thoroughly tested for plane wave excitations. However, the major conclusions of Xiong (1992) on convergence issues should be also valid for controlled source problems. While the physical meaning of the system iterations are 
very clear for general non-symmetric models using equation (A.5), the block partitioning of the matrix $\Gamma^{\prime}$ does not have obvious physical meaning. However, the block iteration of equation (A.14) should be valid as long as it converges.

While the block partitioning of equation (A.6) can be implemented in a straightforward manner, it is more involved to do so for equations (A.14) through (A.17) since we need to keep the memory requirement of the algorithm to only one of the sub-block-matrices. The special features of the unitary matrix $U$ (Tripp, 1982; Tripp \& Hohmann 1984) renders a fast way of partitioning $\Gamma^{\prime}$. For clarity of discussion we write down the form of the unitary matrix $U$ here again:

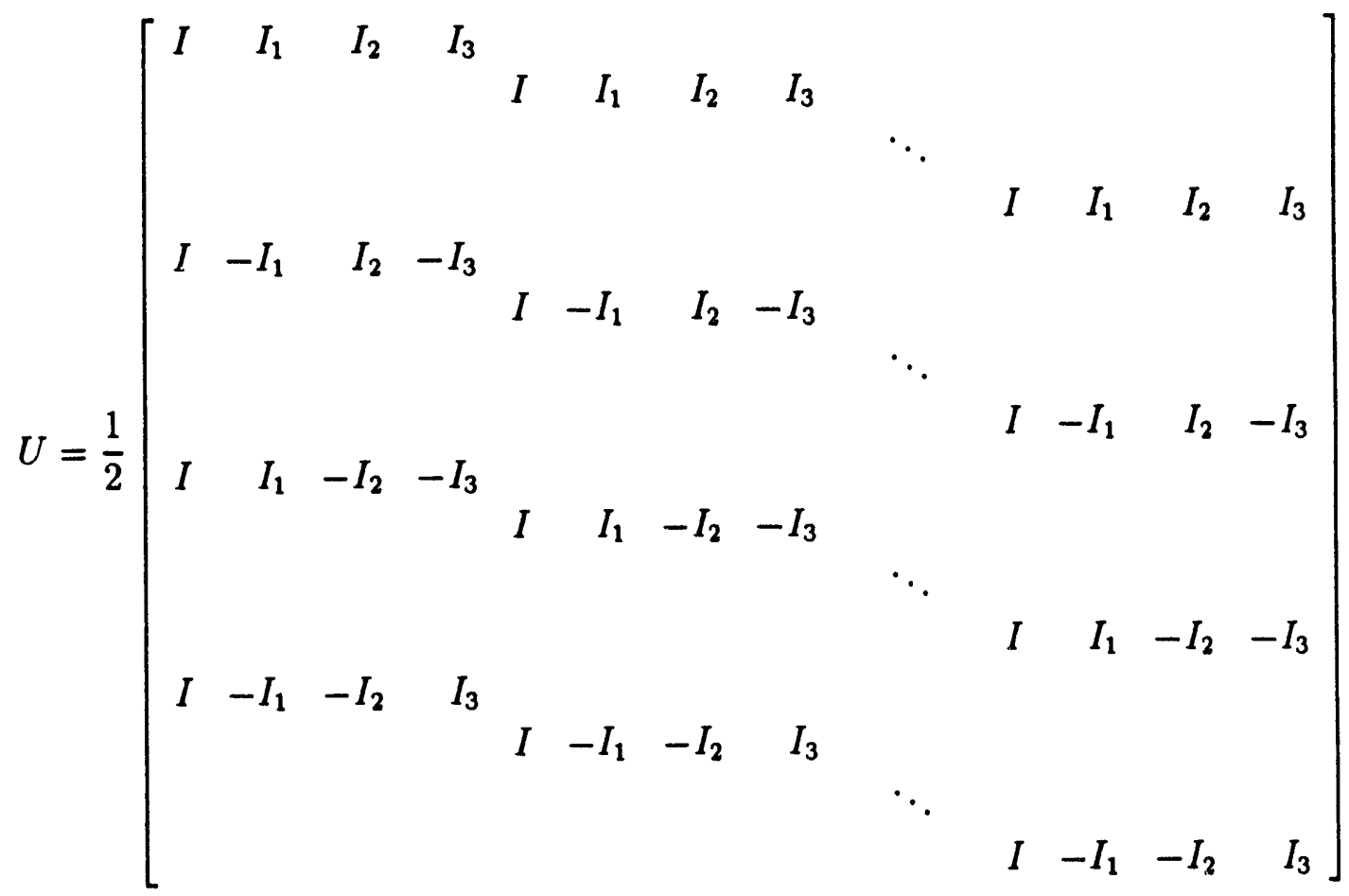

where

$$
\begin{aligned}
& I=\left[\begin{array}{lll}
1 & 0 & 0 \\
0 & 1 & 0 \\
0 & 0 & 1
\end{array}\right] \\
& I_{1}=\left[\begin{array}{rrr}
-1 & 0 & 0 \\
0 & 1 & 0 \\
0 & 0 & 1
\end{array}\right] \\
& I_{2}=\left[\begin{array}{rrr}
-1 & 0 & 0 \\
0 & -1 & 0 \\
0 & 0 & 1
\end{array}\right]
\end{aligned}
$$




$$
I_{3}=\left[\begin{array}{rrr}
1 & 0 & 0 \\
0 & -1 & 0 \\
0 & 0 & 1
\end{array}\right]
$$

All entries not shown in equation (A.18) are zero.

Suppose the total number of cells in one quadrant of the structure is $M$. If we follow the numbering of the quadrants as in Tripp (1982) and Tripp \& Hohmann (1984) and number the matrix entries of $U$ as follows,

$$
\begin{gathered}
i=3 M\left(q_{r}-1\right)+3\left(p_{r}-1\right)+s, \\
j=12\left(p_{c}-1\right)+3\left(q_{c}-1\right)+t \\
p_{r}, \quad p_{c}=1,2, \ldots, M, \\
q_{r}, \quad q_{c}=1,2,3,4, \\
s, \quad t=1,2,3
\end{gathered}
$$

with $i$ representing the number of rows and $j$ the number of columns in $U$, then the elements of $U$ have the property

$$
U_{i j}=\left\{\begin{array}{ll} 
\pm \frac{1}{2} & p_{r}=p_{c} \text { and } s=t \\
0 & p_{r} \neq p_{c} \quad \text { or } s \neq t
\end{array},\right.
$$

where the \pm sign is determined by the values of $p_{r}$, or $p_{c}$, and $s$, or $t$. The meaning of $p_{r}, q_{r}$ etc. are obvious: $p_{r}$ and $p_{c}$ are the numbering for the cells in a quadrant, $q_{r}$ and $q_{c}$ are the numbering for the four quadrants, and $s$ and $t$ are the numbering for the three components.

The elements of the block diagonalized matrix $\Gamma^{\prime}$ as shown in equation (A.10) are

$$
\Gamma_{i j}^{\prime}=\sum_{l} \sum_{k} U_{i l} \Gamma_{l k} U_{j k}
$$

It appears that the block diagonalization procedure requires the full matrix $\Gamma$. However, because of equation (A.28), we can carry out the transforms for a single matrix element in each of the four block matrices associated with the matrix entries in the original matrix $\Gamma$ for the four cells related by group operations, namely, by symmetry relations. Thus if we rewrite $\Gamma_{i j}^{\prime}$ in terms of $p_{r}, q_{r}$ etc., equation (A.29) reduces to

$$
\Gamma_{p_{r} q_{r} s p_{c} q_{c} t}^{\prime}=\sum_{q_{r}^{l}=1}^{4} U_{p_{r} q_{r} s p_{r} q_{r}^{l},} \sum_{q_{c}^{k}=1}^{4} \Gamma_{p_{r} q_{r}^{l} s p_{c} q_{c}^{k} t} U_{p_{c} q_{c} t p_{c} q_{c}^{k} t} .
$$

From the above equation we see that the matrix elements $\Gamma^{\prime}$ for specific cell numbers $p_{r}$ and $p_{c}$ are only related to the matrix elements of $\Gamma$ for symmetry-related cells. This is 
obvious since the transform in equation (A.10) is in fact a transform using symmetrized basis vectors which involves only symmetry-related cells.

Note that on the right hand side of equations (A.29) and (A.30) both $i$ and $j$, or $p_{r} q_{r} s$ and $p_{c} q_{c} t$ vary in the sequence determine by the row index as in equation (A.23). Thus the block diagonalized matrix $\Gamma^{\prime}$ is automatically arranged in the numbering established by equation (A.23) both for the row and for the column. Due to the block diagonal nature of $\Gamma^{\prime}, \Gamma_{p_{r} q_{r} s p_{c} q_{c} t}^{\prime}=0$ for $q_{r} \neq q_{c}$. This also leads to a very important feature of equation (A.30) for our applications: the block partitioning of the matrix $\Gamma^{\prime}$ induced by dividing a quadrant into substructures results in four identically partitioned block matrices for $q_{r}=q_{c}=1,2,3$, and 4, i.e., the four equations (A.14) through (A.17) are identically partitioned. We can divide one quadrant into arbitrary substructures by numbering $p_{r}$ and $p_{c}$ the way we like. This also allows us to store the partitioned sub-block-matrices in two files per quadrant, i.e., for each of the four block matrices $\Gamma^{1}$ through $\Gamma^{4}$, with one file for the diagonal sub-block-matrices and one file for the off-diagonal sub-block-matrices. This enables the fastest possible data swap between the computer memory and the hard disk.

\section{A.3.4 Convergence tests}

The block partitioning of the equations (A.14) through (A.17) enabled by equation (A.30) results in convergent block iterative algorithms for the test studies that we carried out so far. While a rigorous theoretical proof of convergence is still missing, numerical tests with realistic models may shed some light on the behavior of the proposed method.

For our test studies we use a geothermal reservoir model proposed by Pellerin (1992) and Pellerin et al. (1993) which is shown in Figure 1. The model consists of two block conductors, one of dimensions $6 \times 4.8 \times 0.375 \mathrm{~km}^{3}$ with $5 \Omega \cdot \mathrm{m}$ resistivity representing a clay cap, and the other of dimensions $2.7 \times 2.7 \times 5.4 \mathrm{~km}^{3}$ with $25 \Omega \cdot \mathrm{m}$ resistivity respresenting a geothermal reservoir. The transmitter is a bipole current source of $1 \mathrm{~km}$ along the $x$-direction with the center of the bipole located at $x=0$ and $y=-7400 \mathrm{~m}$. Note that the $y$-coordinate of the bipole center is siightly different from that of Pellerin (1992) and Pellerin et al. (1993).

We have computed the responses of this model with various ways and various numbers of discretizations. For various divisions of the body with 2 to 10 substructures the block iterations converged in about 3 to 9 iterations for high frequencies and 10 to 40 iterations for low frequencies. The number of iterations needed for convergence depended mostly on the number of substructures. Increasing the numbers of cells in all substructures while keeping the number of substructures unchanged resulted in slight increases in the numbers of iterations needed for convergence. The block iterations converged no matter how the body was divided into substructures. 
Table 1 shows the numbers of iterations needed for convergence and approximate run times for solving the matrix equations for three divisions of body with 4,7 , and 10 substructures as well as the approximate run time needed by direct solutions of the four equations (A.14) through (A.17). The model was discretized into 498 cells per quadrant, with 369 cells for the clay cap and 129 cells for the reservoir. The numbers of iterations shown in Table 1 are averaged for the four equations (A.14) through (A.17). The program was run on a Sun SPARCstation 10/30.

That the number of iterations needed for convergence increases for low frequencies may be explained by the fact that the condition numbers of the systems increase as the frequency decreases. Physically speaking, this corresponds to increased coupling between the substructures at low frequencies. The condition numbers of the scattering matrices also increase with the conductivity contrast of a conductor with respect to the conductivity of the host medium. The conductivity contrasts of the reservoir model are 40 for the clay cap and 8 for the reservoir. Those are fairly low contrasts, a reason why our algorithms converged so fast.

The block iterations using hard disks usually take a large amount of CPU time for data swapping between the computer core memory and the hard disks. For our applications we found that the ratio of the CPU time spent on data movement to the CPU time for computations were about 2 to 2.5 for each ileration. Thus each iteration actually requires about 3 to 3.5 times the CPU time needed for floating point computations. Since the conventional Gauss-Seidel iterative method requires about the same amount of computation time as the direct solution with $L U$ decomposition for the number of iterations equal to one third of the dimension of the matrix, our block iterative method will save computation time if solutions converge within a number of iterations equal to $1 / 3.5$ times the number of cells, or 142 iterations for the reservoir model with 498 cells, if we ignore the computation times required by the factorizations of the diagonal sub-blockmatrices. In our examples, due to the reduced time for factorizing the sub-block-matrices for 7 and 10 substructures, we see that the total computation time is reduced the most for 7 and 10 substructures, even though the numbers of iterations are increased. However, if we compare the total times required at $0.00{ }^{\prime} 85 \mathrm{~Hz}$ for 7 and 10 substructures, we see thac using more substructures actually required more computation time due to the increased number of iterations. Therefore, there is a optimal number of substructures for each application which depends on the number of cells as well as the condition numbers of the systems. If our block iterative method is implemented on a parallel machine, the CPU time needed by the data movements will be saved since the sub-block-matrices can be stored in local processing units, and the efficiency of our method will be increased.

In order to show the validity of our method, we finally present a comparison with a finite difference time domain (FDTD) solution for this geothermal reservoir model. The FDTD results were computed by Kriegshauser (personal communication) using the 
Druskin-Knizhnerman program (Druskin \& Knizhnerman, 1988) compliments of the University of Köln, Köln, Germany.

Figure 2 shows the comparisons of the voltages due to $\partial B_{z} / \partial t$ at receivers located at $y=0$, and $1750 \mathrm{~m}$ computed by our algorithm and by FDTD. The two solutions obvious agree in general.

Table 1 : Computation time (in seconds) for different number of substructures. The discretizations are the same with 498 cells per quadrant. "Factors" refers to the ratio of time needed by direct solutions to the total times needed by the block iterative method. The numbers of iterations are averagaed for the four equations (A.14) through (A.17).

\begin{tabular}{c|c|c|c|c|c|c}
\hline \multirow{2}{*}{} & \multirow{2}{*}{ Iterations } & \multicolumn{3}{|c|}{ Times } & \multirow{2}{*}{ Factors } \\
\cline { 3 - 7 } & & Factorization & Iteration & Total & \\
\hline \multicolumn{2}{c|}{ Direct solution } & & 7423 & & 7423 & 1 \\
\hline \multirow{4}{*}{$\begin{array}{c}4 \\
\text { Substructures }\end{array}$} & $1118.5 \mathrm{~Hz}$ & 8 & 930 & 507 & 1437 & 5.2 \\
\cline { 2 - 7 } & $1.1185 \mathrm{~Hz}$ & 24 & 930 & 1385 & 2315 & 3.2 \\
\cline { 2 - 7 } & $.00185 \mathrm{~Hz}$ & 26 & 930 & 1504 & 2434 & 3.0 \\
\hline \multirow{4}{*}{$\begin{array}{c}7 \\
\text { Substructures }\end{array}$} & $1118.5 \mathrm{~Hz}$ & 9 & 245 & 514 & 759 & 9.8 \\
\cline { 2 - 7 } & $1.1185 \mathrm{~Hz}$ & 26 & 245 & 1379 & 1624 & 4.6 \\
\cline { 2 - 7 } & $.00185 \mathrm{~Hz}$ & 29 & 245 & 1500 & 1745 & 4.3 \\
\hline \multirow{4}{*}{$\begin{array}{c}\text { Substructures } \\
10\end{array}$} & $1118.5 \mathrm{~Hz}$ & 9 & 105 & 530 & 545 & 13.7 \\
\cline { 2 - 7 } & $1.1185 \mathrm{~Hz}$ & 26 & 105 & 1390 & 1495 & 5.0 \\
\cline { 2 - 7 } & $.00185 \mathrm{~Hz}$ & 34 & 105 & 1825 & 1840 & 4.0 \\
\hline
\end{tabular}




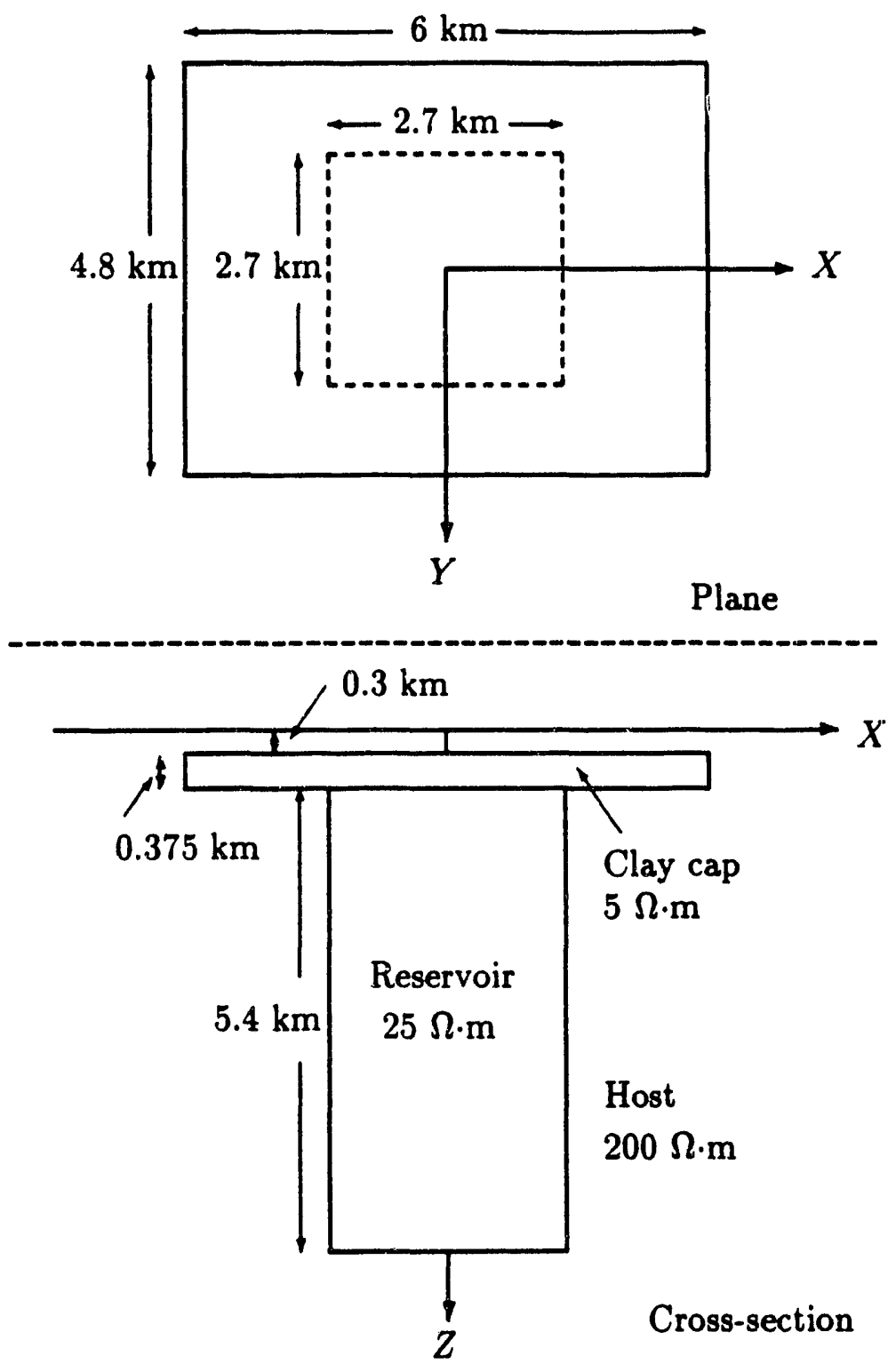

Figure 1. A geothermal model consisting of a clay cap of $5 \Omega \cdot \mathrm{m}$ and a reservoir of $25 \Omega \cdot 11$. 


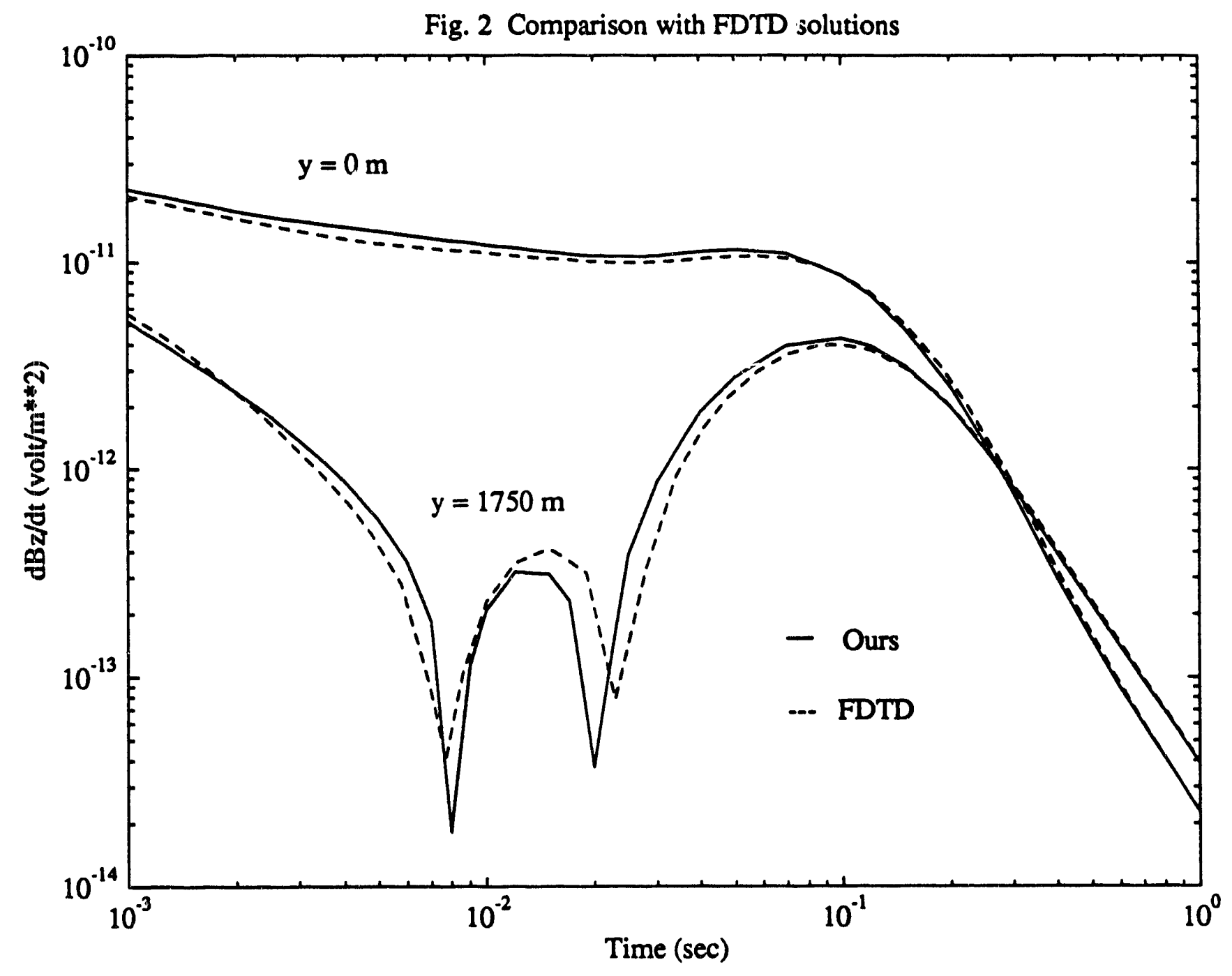




\section{A.4 Comparisons with Program EM3D}

We present some comparisons with the integral equations modeling program EM3D developed by ihe CEMI (Consortium for EM Modeling and Inversion) group of University of Utah. The name of our program is SYSEM.

\section{A.4.1 Cumputation time comparisons}

Table 2 shows computation time comparisons for EM3D and SYSEM for a 3D body buried in a 5 layered earth with a rectangular loop excitation with 13 receivers at the earth's surface for one frequency. The low contrast version of EM3D was used in the computations. Program SYSEM was run with an accuracy level of 1 . It is anticipated that the low contrast version of EM3D can only match the accuracy level 1 of SYSEM. The body was not further divided into substructures in the computations. Three different quadrant discretizations, $4 \times 4 \times 1,8 \times 8 \times 1$, and $14 \times 14 \times 1$ were tested. Group reductions were used for all the computations since the models we ran are symmetric. The cells for every discretization had the same size. The programs were run on Sur SPARCstations. The discretizations of $4 \times 4 \times 1$ and $8 \times 8 \times 1$ were run on a SPARCstation 1 , and the discretization of $14 \times 14 \times 1$ was run on a SPARCstation 2. Both programs were compiled with an optimization option of -03 with the F77 compiler.

SYSEM was run both with and without spatial symmetry reduction in order to test the general performance of the program. For the discretization of $4 \times 4 \times 1$, SYSEM ran more than 30 time faster than EM3D. The factor decreases as the number of cells increases. This is mostly due to the fact that the computation time needed to solve the matrix equation increases cubically with the number of cells and this portion of the computation is standard and hardly optimizable except for using vector computations. The vectorizable percentages listed in Table 2 show the percentage of computation time needed to solve the matrix equation and to block diagonalize the matrices. This percentage increases with the number of cells. From Table 2 we see that program SYSEM with spatial symmetry reduction is highly vectorizable for the discretization of $14 \times 14 \times 1$. The vectorizable percentage increases rapidly with the number of cells for SYSEM. With a discretization of $16 \times 16 \times 1$ (not shown in the table) the vectorizable percentage reaches $90 \%$ for SYSEM with spatial symmetry reduction. Thus the ratio EM3D/SYSEM will increase if the programs are run on computers with vector facilities. The high efficiency of SYSEM compared with EM3D for small numbers of cells may indicate that EM3D has too much overhead. If we compare the computation times for the discretization of $14 \times 14 \times 1$ cells less the times required by the solutions of the matrix equations, which about is 3100 seconds for EM3D, and about 460 seconds for SYSEM without using the spatial symmetry reduction, we see that SYSEM is about 6.7 time faster than EM3D in general for the computations 
involved in forming the matrix and in computing secondary fields. This is a reasonable speed-up using analytical integrations in the vertical direction for simple models, and this speed-up can be much higher for high contrast and complex models when high accuracy for the numerical integrations of the Green's functions is necessary. The discretizations shown in Table 2 all used equal-size cells, so that the scattering matrices were symmetric. It appeared to us that EM3D does not make use of this symmetry property of the matrices and thus solutions of the matrix equations required by EM3D took more than twice the time required by SYSEM using direct methods.

Table 2 : Comparisons of computation time (in seconds) required by EM3D and SYSEM. Group reductions are used and the discretizations are for one quadrant only. SS stands for spatial symmetry.

\begin{tabular}{|c|c|c|c|c|c|}
\hline \multicolumn{3}{|c|}{ Discretizations } & $4 \times 4 \times 1$ & $8 \times 8 \times 1$ & $14 \times 14 \times 1$ \\
\hline \multirow{2}{*}{\multicolumn{2}{|c|}{ EM3D }} & Time & 959.4 & 2410.4 & 4321.7 \\
\hline & & Vectorizable Percentage & 0.3 & 8 & 28 \\
\hline \multirow{6}{*}{ SYSEM } & \multirow{3}{*}{$\begin{array}{c}\text { With } \\
\text { SS Re- } \\
\text { duction }\end{array}$} & Time & 28.6 & 115.9 & 722.2 \\
\hline & & Vectorizable Percentage & 8 & 45 & 80 \\
\hline & & Factor (EM3D/SYSEM) & 33.5 & 20.8 & 6.0 \\
\hline & \multirow{3}{*}{$\begin{array}{l}\text { Without } \\
\text { SS Re- } \\
\text { duction }\end{array}$} & Time & 31.8 & 178.8 & 1086.7 \\
\hline & & Vectorizable Percentage & 7 & 29 & 57 \\
\hline & & Factor (EM3D/SYSEM) & 30.2 & 13.5 & 4.0 \\
\hline
\end{tabular}




\section{A.4.2 Comparisons of high contrast solutions}

Due to the overall high accuracy of the program, SYSEM can model conductive models with conductivity contrasts of up to 10000 without using special basis functions, and thus can be used for arbitrarily shaped high contrast models.

Figure 3 shows a model used by Newman \& Hohmann (1988). It is a brick model of $800 \times 25 \times 100 \mathrm{~m}^{3}$ with a resistivity of $0.1 \Omega \mathrm{m}$ buried $100 \mathrm{~m}$ deep in a two layered earth. The upper layer of the earth is $50 \mathrm{~m}$ thick with a resisitivity of $10 \Omega \mathrm{m}$. The conductivity of the basement is variable. The transmitter is a fixed loop of $500 \mathrm{~m}$ on a side with its near wire located at $y=-250 \mathrm{~m}$.

Figure 4 shows the comparisons of the voltages due to $\partial B_{z} / \partial t$ at a receiver located at $y=-80$ for two basement resisitivities, $100 \Omega \cdot \mathrm{m} 1000 \Omega \cdot \mathrm{m}$. Thus the contrasts of the model are 1000 and 10000 . Overall agreement for the solutions with $100 \Omega \cdot \mathrm{m}$ basement is obvious. Only the late time decays show a slight difference. Our solution decays faster than the solution of Newman \& Hohamnn. This difference appears much larger for the solutions with a $1000 \Omega \cdot m$ basement case. Newman \& Hohmann also computed a 10000 $\Omega \cdot m$ basement case. Our solution for that case does agree with Newman \& Hohmann in the amplitudes of peak anomalies, but reverses the sign at a much earlier time, approximately $0.8 \mathrm{msec}$, whereas the solution of Newman \& Hohmann reverses sign at about 2.2 insec. Considering that the contrast for the basement resistivity of $10000 \Omega \cdot \mathrm{m}$ may exceed the designed accuracy of SYSEM, we do not show the solutions for that case. We computed the solutions with a couple of discretizations, $10 \times 1 \times 8$ per quadrant, $20 \times 1 \times 10$ per quadrant, and $20 \times 1 \times 15$ per quadrant. The solutions converged.

\begin{tabular}{|c|c|c|}
\hline $10 \Omega \cdot \mathrm{m}$ & $50 \mathrm{~m}$ & \\
\hline $\begin{array}{l}100 \Omega \cdot \mathrm{m} \\
\& \\
1000 \Omega \cdot \mathrm{m}\end{array}$ & & $\begin{array}{l}0.1 \Omega \cdot \mathrm{m} \\
\mathrm{t}=25 \mathrm{~m} \\
\mathrm{SL}=800 \mathrm{~m} \\
\mathrm{DE}=100 \mathrm{~m} \\
\mathrm{DB}=100 \mathrm{~m}\end{array}$ \\
\hline
\end{tabular}

Figure 3. A high contrast model 
Fig. 4 Comparison with Newman \& Hohmann

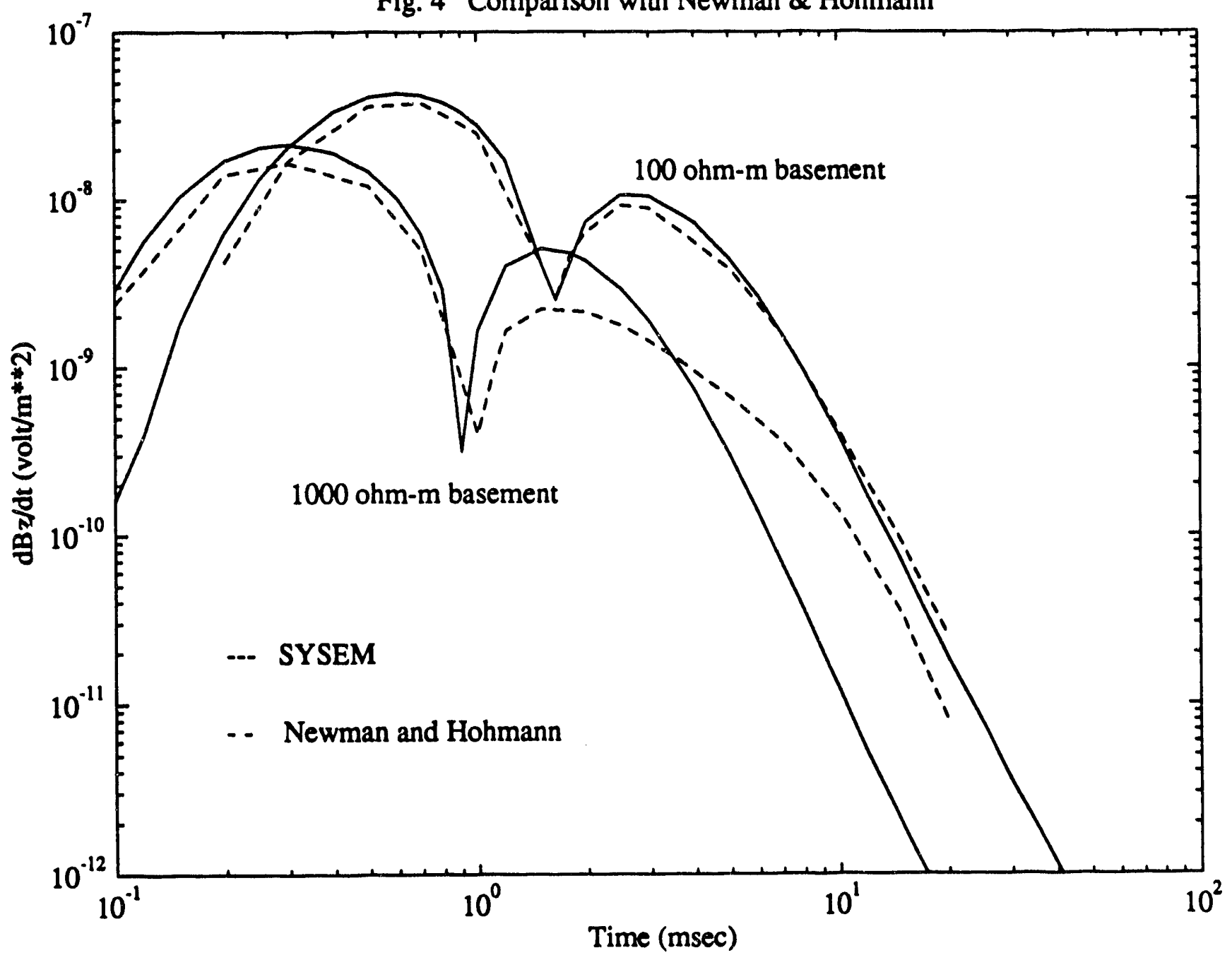




\section{A.5 References}

Christensen, N.B., 1990. Optimized fast Hankel transform filters, Geophysical Prospecting, 38, 545-568.

Druskin, V.L., and Knizhnerman, L.A., 1988, A spectral semidiscrete method for the numerical solution of 3-D nonstationary problems in electrical prospecting: Phys. Sol. Earth, 24, 641-648.

Hohmann, G.W., 1971. Electromagnetic scattering by conductors in the Earth near a source of current, Geophysics, 36, 101-131.

-, 1975. Three-dimensional induced polarization and electromagnetic modeling, Geophysics, 40, 309-324.

Pellerin, L.D., 1992, Application of 3D modeling in EM exploration, Ph.D dissertation, University of Utah.

Pellerin, L.D., J.M. Johnston and G.W. Hohmann, 1993, Evaluation of electromagnetic methods in geothermal exploration, Geophysics, submitted.

Tripp, A.C., 1990. Group theoretic reduction of the electromagnetic impedance matrix for large-contrast symmetric prisms in a layered earth, PAGEOPH, 133, 127-147.

-, 1982, Multidimensional electromagnetic modeling, Ph.D thesis, University of Utah.

Tripp, A.C. and G.W. Hohmann, 1984, Block diagonalization of the electromagnetic impedance matrix of a symmetric buried body using group theory: IEEE Trans. on Geoscience and Remote Sensing, GE-22, 62-68.

Weidelt, P., 1975. Electromagnetic induction in three-dimensional structures: J. Geophysics, 41, 85-109.

Xiong, Z., 1992, Electromagnetic modeling of three-dimensional structures by the method of system iteration using integral equations: Geophysics, 57, 1556-1561.

-, 1991, Electromagnetic modeling of three-dimensional structures in stratified anisotropic earths using the integral equation method: in U. Schmucker and A. Kirsch, Entwicklung und Erprobung von Algorithmen zu dreidimenisonalen Modellrechnungen in der erdmagnetischen Tiefensondierung, report for project Schm 101/15-1, Univeristät Göttingen.

-, 1990. Umkehraufgaben bei 3D elektromagnetischen Induktionsproblemen in geophysikalischen Anwengdungen, Dissertation, Universität Göttingen. 
,- 1989. Electromagnetic fields of electrical dipoles embedded in a stratified anisotropic earth, Geophysics, 54, No. 12, 1643-1646. (Errata in V. 56, 414: the $k_{i}^{ \pm}$in eq. (28) and (39) should be $u_{i}^{ \pm}$and $v_{i}^{ \pm}$, respectively).

Xiong, Z. \& A.C. Tripp, 1993, Scattering matrix evaluation using spatial symmetry in electromagnetic modeling, Geophysical Journal International, to appear.

Xiong, Z., Y., Luo, S. Wang and G. Wu, 1986, Induced-polarization and electromagnetic modeling of a three-dimensional body in a two-layer anisotropic earth: Geophysics, 51, 2235-2246. 


\section{Appendix B}

\section{Focused arrays}

\section{or \\ Improving the resolution of the inverse problem by adaptive data weighting.}

1. The geo-electric inverse problem is ill-posed, which leads to a large class of models which match the data. In many cases, the difference between these models can be very significant. Hence, decreasing the size of this class for a particular application can be of great importance.

Increasing the number of observation points can reduce the interpretation ambiguity, but at the cost of increased ccmputational time. An increased number of observations can also increase the need for regularization, which if done haphzzardly could obviate any advantage gained through the additional data. It seems like a better policy to actually design the data set to be consistent with the best estimate of the geology at the very beginning. The present work discusses the theoretical framework for fully utilizing data information and a-priori model information via data weighting.

In order to use only valuable information we transform data using an appropriate scaling or weighting matrix. Hence, instead of giving all data equal significance, we emphasize those linear combinations of data which contain the most information. Besides reducing the dimension of the inverse problem matrices, this should lead to a reduction 
of the inversion model ambiguity. The computer trials of this technique are currently underway [1].

As a measure of the difference in detectibility of two different models we take the $l^{2}$ norm of their vectorial difference. We wish to find a data weighting which maximizes this difference.

Let $\gamma$ and $\gamma+\sigma$ be the conductivities of the host medium and the medium with an inclusion, respectively. Let us inject a current $j=\left\{j_{k}\right\}$ at the points $k=1,2, \ldots, n$. The corresponding potentials, measured on the surface, are $u$ for the host medium and $w$ for the medium with an inclusion; they are related to the currents through impedance matrices $Z_{\gamma}$ and $Z_{\gamma+0}$ as

$$
u=Z_{\gamma} j
$$

and

$$
w=Z_{\gamma+\sigma} j
$$

Injecting a current $j_{k}$ at each transmitter current electrode $k$ and measuring a voltage $w(i)$ at receiver electrode $i$, for $i=1,2, \ldots l$, corresponds to the so-called pole-pole array. We introduce focused arrays using some weighting matrix $W$. This weights the intensities of the currents injected at the transmitter electrodes in order to maximize the difference in the response voltages for a host $\mathbf{n}$ dium and a medium with an inclusion. In other words we would like to design arrays which focus most of the currents to the region of the inclusion.

2. We introduce here the mathematical description of the problem. We consider the case of direct current injection. Let a domain $\Omega$ be a region of the Earth, on the surface $\partial \Omega$ of which is applied a current $f$. Every current $f$ on the surface can be represented as a weighted sum of injected currents $j_{k}$.

$$
f=\sum_{k=1}^{n} \alpha_{k} j_{k}
$$

Normalizing the currents introduces the additional requirement that

$$
\sum_{k=1}^{n} \alpha_{k}^{2}=1
$$

The potentials and currents for the host medium $\gamma$ and the perturbed medium $\gamma+\sigma$ satisfy the conductivity equations

$$
\nabla \cdot(\gamma+\sigma) \nabla w=0 \text { in } \Omega,(\gamma+\sigma) \frac{\partial w}{\partial n}=f \text { on } \partial \Omega
$$

and

$$
\nabla \cdot \gamma \nabla u=0 \text { in } \Omega, \gamma \frac{\partial u}{\partial n}=f \text { on } \partial \Omega
$$


We consider the norm of the difference between response voltages $w$ and $u$ on the surface for the applied current $f$ to be a measure of information content of the specific data set, associated with the current $f$. Thus the measure is

$$
I=\|w-u\|_{L_{2}(\partial \Omega)}=\int_{\partial \Omega}(w-u)^{2}
$$

The array which focuses maximal current to the vicinity of the inclusion corresponds to the current which maximizes the response from the inclusion, i.e. maximizes the value I.

$$
M=\max _{f} I(f)=\max _{f}\|w-u\|_{L_{2}(\partial \Omega)}
$$

The problem (B.8) is an eigenvalue problem which has been considered in connection with the problem of distinguishability in a number of papers (see, for ex. $[2,3,4,5,6]$ ).

In previous notation the functional (B.8) is represented as:

$$
M=\max _{\left\{\alpha_{k}\right\}}\left\|Z_{\gamma+\sigma} f-Z_{\gamma} f\right\|=\max _{\left\{\alpha_{k}\right\}}\left\|\left(Z_{\gamma+\sigma}-Z_{\gamma}\right) \sum_{k=1}^{n} \alpha_{k} j_{k}\right\|
$$

In this expression $j_{k}$ is a vector with components equal to the Kronecker delta function, $j_{k}(i)=\delta_{i k}$, it describes the unit current injected at the point $k$. Since the function $w$ is measured only at a finite number of points, we change the norm in (B.9) to the euclidean sum of squares.

We now require that the coefficients $\alpha_{k}$, subject to the restriction (B.4), satisfy expression (B.9). These coefficients are found numerically by various methods of solving eigenvalue problems [7].

The values $M_{i}$ and corresponding currents $J_{i}=\sum_{k} \alpha_{k}^{i} j_{k}$ which satisfy (B.9) form the eigensystem of the problem (B.9); these currents are orthogonal. The current $J_{1}$, which corresponds to the maximal eigenvalue $M_{1}$, gives the most amount of information about the inclusion. In general, the currents $\left\{J_{i}\right\}$ form a sequence, corresponding to monotonical decreasing values $M_{i}$ of the functional (B.8). In practice our measurements always have some error $\epsilon_{0}$. Therefore, if the response voltages on the surface differ by less than $\epsilon_{0}$, we cannot distinguish them, and the corresponding currents do not give any useful information. Hence, we need to consider only the data from the currents $J_{i}$, which provide a value of $M_{i}$ which is greater than $\epsilon_{0}$. Let the number of them be $N$. Suppose the voltage difference data set is generated by an arbitrary current $f$, such that

$$
f=\sum_{i=1}^{N} \kappa_{i} J_{i}
$$

where $\kappa_{i}$ are the coefficients of the expansion of the current $f$ in terms of the system of the currents $\left\{J_{i}\right\}$. Using the property of orthogonality of eigencurrents in the expression 
(B.7), the information content of such a set of data can be estimated as [6] :

$$
\mathcal{I}^{2}(f)=\sum_{i=1}^{N} \kappa_{i}^{2} M_{i}^{2}
$$

3. Lets now discuss the use of boundary measurements for determining the parameters of the inclusion. In order to consider the data that we have altogether, we form "long" vectors $I$ and $v$, composed of he vectors of the currents $j_{k}$ and corresponding voltage differences $w-u$,

$$
I=\left(\begin{array}{c}
j_{1} \\
j_{2} \\
\ldots \\
j_{n}
\end{array}\right), \quad v=\left(\begin{array}{c}
(w-u)_{1} \\
(w-u)_{2} \\
\ldots \\
(w-u)_{n}
\end{array}\right)
$$

where $n$ is the number of currents injected, and $(w-u)_{k}$ is a vector of voltage differences caused by injection of the current $j_{k}$.

The transformation described in the previous section rotates the vector $I$ to the vector $\tilde{I}$, using the weight matrix $W$ composed of the coefficients $\alpha_{k}^{i}$ :

$$
\tilde{I}=W I, \quad \tilde{I}=\left(\begin{array}{c}
J_{1} \\
J_{2} \\
\ldots \\
J_{n}
\end{array}\right), \quad W=\left(\begin{array}{cccc}
\alpha_{1}^{1} E & \alpha_{2}^{1} E & \ldots & \alpha_{n}^{1} E \\
\alpha_{1}^{2} E & \alpha_{2}^{2} E & \ldots & \alpha_{n}^{2} E \\
\ldots & \ldots & \ldots & \ldots \\
\alpha_{1}^{n} E & \alpha_{2}^{n} E & \ldots & \alpha_{n}^{n} E
\end{array}\right)
$$

where $E$ is an identical matrix.

Simultaneously under this transformation the vector $v$ becomes the vector $\tilde{v}$ :

$$
\tilde{v}=W v,
$$

so that due to (B.9) every $n$ subsequent "vector components" of the "long" vector $\tilde{v}$ have a norm which is greater than that of the remaining $n$-sets. Since only first $N$ eigencurrents corresponding to noticeable values of the voltage difference are useful, we can cut all other components, which provide negligible information. This reduces the dimension of the problem. A possible approach to the inversion problem is to consider only one eigencurrent on the first iteration, and include additional currents, as the error of the least squares approximation decreases. As a result of such a cut we obtain the vectors of least dimension, $\hat{I}$ and $\hat{v}$.

4. Now we describe the effect of using only $N$ eigencurrents on the solution of the inverse problem.

Suppose that the initial conductivity model is described by parameters $\sigma_{1}, \ldots \sigma_{m}$. The real body, however, possesses a different conductivity described by the vector $\sigma_{1}+$ $\Delta \sigma_{1}, \ldots, \sigma_{m}+\Delta \sigma_{m}$. We want to find the vector of corrections $\Delta \sigma=\left(\Delta \sigma_{1}, \ldots, \Delta \sigma_{m}\right)$ from the measurements of the voltage differences. 
The solution of this inverse problem is traditionally formulated as a sequence of linearized least squares problems :

$$
\|A x-\hat{v}\| \rightarrow \min _{x}
$$

where $x$ is a vector of corrections of parameters of the inclusion, $x=\Delta \sigma$, and $A$ is a $p$ by $m$ matrix of data derivatives, which describe the change of the measured values of potential (if the current $\hat{I}$ were injected ) in comparison with the starting model, $p=N \cdot l$ :

$$
A=\left\{a_{i j}\right\} ; \quad a_{i j}=\frac{\partial \hat{v}_{i}}{\partial \sigma_{j}} .
$$

Consider the singular value decomposition of the matrix $A$

$$
A=U \Sigma V^{T}
$$

where $U$ and $V$ are orthogonal matrices of left $\xi_{i}$ and right $\zeta_{i}$ singular vectors, and $\Sigma$ is a diagonal matrix of singular values $\lambda_{i}$ of the matrix $A$. If the matrix $A$ is a square matrix, $\lambda_{i}, \xi_{i}$, and $\zeta_{i}$ are its eigenvalues and eigenvectors.

The vectors $x$ and $\hat{v}$ have the following representation through the singular values $\lambda_{i}$ and the singular vectors $\xi_{i}$ and $\zeta_{i}$ of the matrix $A$ :

$$
\hat{v}=\sum_{i=1}^{m} \beta_{i} \xi_{i}, \quad x=\sum_{i=1}^{m} x_{i} \zeta_{i}, \quad A x=A\left(\sum_{i=1}^{m} x_{i} \zeta_{i}\right)=\sum_{i=1}^{m} \lambda_{i} x_{i} \xi_{i} .
$$

where $\beta_{i}$ and $x_{i}$ are the projections of the vectors $\hat{v}$ and $x$ on the directions $\xi_{i}$ and $\zeta_{i}$, respectively.

Suppose that the error is uniformly distributed among the measured data, so it can be represented as a ball of uncertainty in a space of data. This implies that the error in the data is distributed uniformly along the components $\beta_{i}$. Using the last expansion one can show that the choice of the vector $\hat{v}$ with maximal norm provides the best possible resolution for the components of the unknown vector $x$. Suppose that every component of the data vector $\beta_{i}$ has a disturbance $\epsilon$. Let $\eta_{i}$ be the error arising in the $i$-th component of the solution $x$ due to the error in the data $\hat{v}$. Then

$$
\beta_{i}+\epsilon=\lambda_{i}\left(x_{i}+\eta_{i}\right)
$$

and

$$
\sum_{i=1}^{m} \frac{x_{i}^{2}}{\eta_{i}^{2}}=\frac{1}{\epsilon^{2}} \sum_{i=1}^{m} \beta_{i}^{2}
$$

The right hand side of this expression equals the squared norm of the vector $\hat{v}$, divided by $\epsilon^{2}$. Thus maximizing $\hat{v}$ maximizes the ratio between the true values of the unknown vector parameters and the error in its determination caused by noise in data. 
Another effect that increases the resolution is connected with rotating the eigendirections of the matrix $A$. This result is a marked improvement over what might be expected for SVD inversion, as we will now demonstrate.

Consider the case where the matrix $A$ is ill-posed and the data of measurements contain some error. In this case, suppression of the singular values below the error level stabilizes the least squares solution and hence is used often in inversion. In the general case for arbitrary data which do not necessarily satisfy (B.9), neglecting the smallest singular values may lead to an increase of stability of the solution, but it may cut the components of the vector $x$ which are far from being small. When we use the data of weighted currents, we maximize the norm of the vector $\hat{v}^{2}$, and this means we maximize the product

$$
(\lambda \cdot x)^{2}=\sum_{i}\left(\lambda_{i} x_{i}\right)^{2} .
$$

Since the vector $x$ is fixed, this product is maximized only if the larger values $\lambda_{i}$ correspond to the larger components $x_{i}$ of the vector $x$, and smaller values $\lambda_{i}$ correspond to the smaller components. Therefore when small $\lambda_{i}$ are neglected the perturbation on the solution will be as small or smaller than that introduced by the truncation in the case of untransformed data, which again demonstrates that the transformed data gives optimal solution resolution. 


\section{Bibliography}

[1] Cherkaeva,E. and Tripp,A.C., 1993, Adaptive focused current electrical imaging in Geophysics: in preparation.

[2] Cherkaeva,E. and Kohn,R., 1992, Distinguishability of inclusions in the presence of noisy measurementc: in preparation.

[3] Dobson,D.C., 1992, Estimates on resolution and stabilization for the linearized inverse conductivity problem: Inverse problems, 4.

[4] Gisser D.G., Isaacson,D. and Newell J.C , 1990, Electric current computed tomography and eigenvalues: SIAM J. Appl.Math., 50, 1623-1624.

[5] Isaacson,D., 1986, Distiuguishability of conductivities by electric current computed tomograpky: IEEE Trans. Med. Imaging MI-5, 91-95.

[6] Isaacssn,D. and Cheney,M., 1989, Current problems in impedance imaging: in Inverse Problems in Partial Differential Equations, ed. D.Colton, R.Ewing and W.Rundell.

[7] Isaacson,E. and Keller H.B., 1966, Analysis of Numerical Methods: Wiley, New York. 

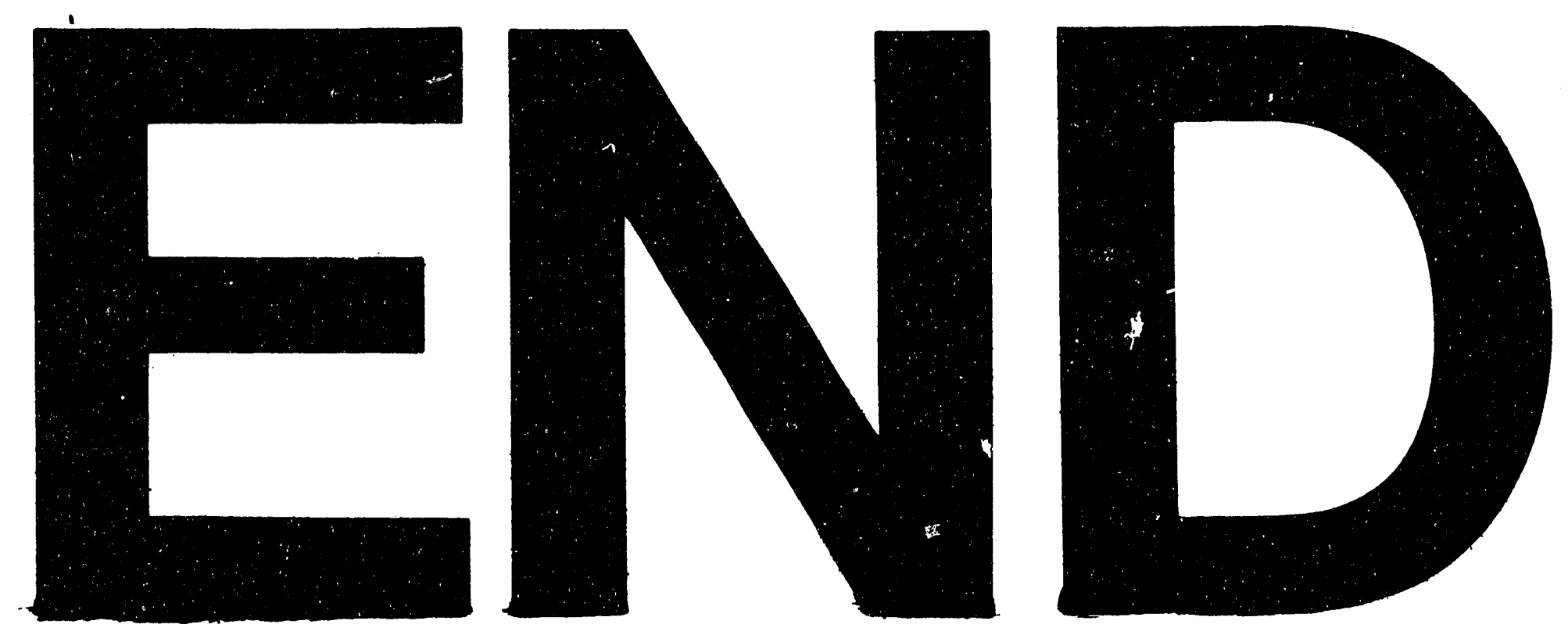

$x$

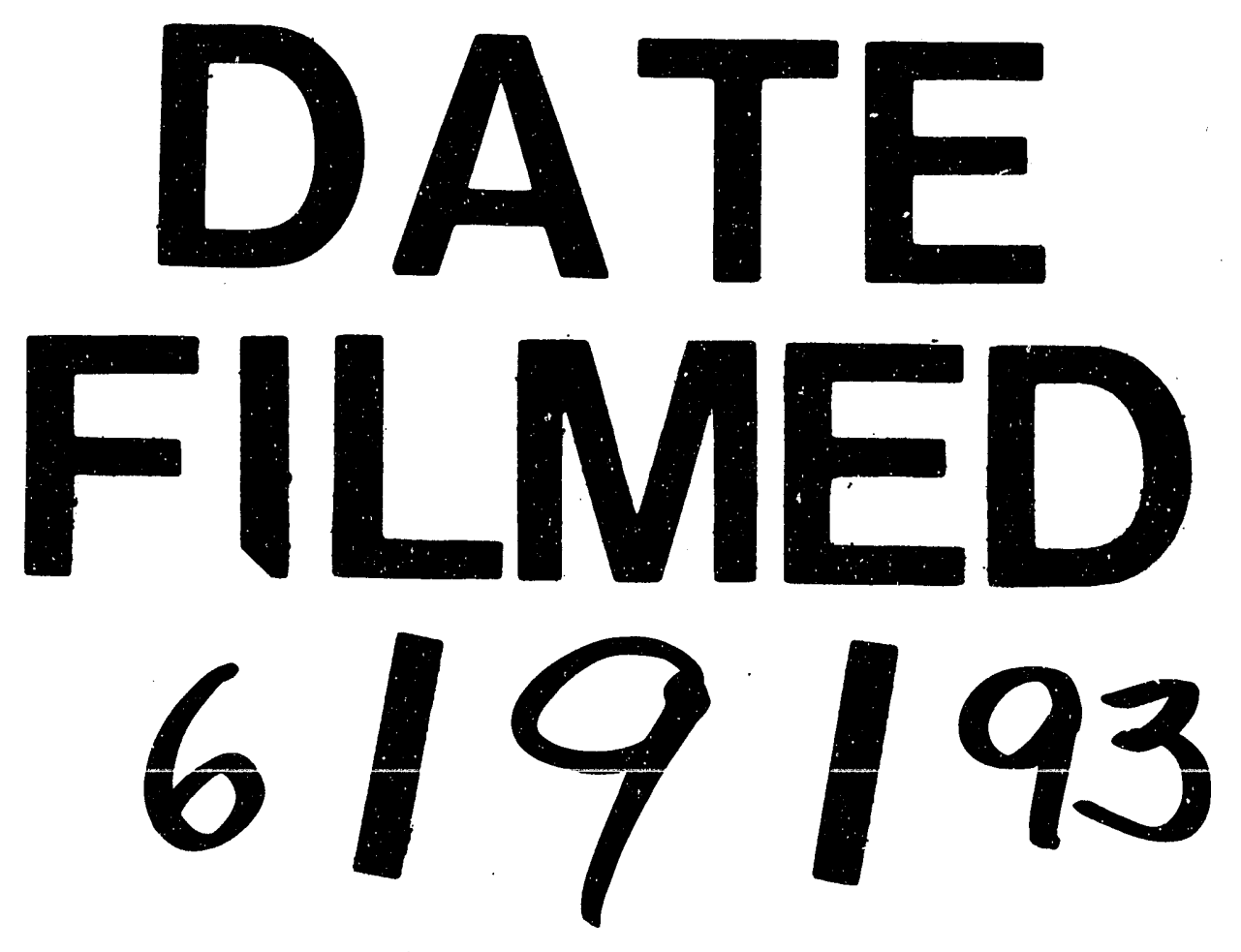


